\title{
Operando Transmission Electron Microscopy of Noble Metal Nano-catalysts During CO Oxidation
}

See Wee Chee ${ }^{1,2}$ and Utkur Mirsaidov ${ }^{1,2}$

1. Department of Physics, National University of Singapore, Singapore

2. Center for Bio-Imaging Sciences, Department of Biological Sciences, National University of Singapore, Singapore

* Corresponding author: mirsaidov@nus.edu.sg

Metal nanoparticles are common catalyst materials for a broad range of chemical reactions in industry. However, the current challenge in optimizing the performance of these nanoparticle catalysts is that we have limited insights into the "working state" of these materials. Nano-catalysts commonly re-structure under reaction conditions and their catalytic performance is determined by these dynamical structures. Conventional high-resolution characterization techniques cannot probe these structures because they typically require a vacuum environment. Hence, significant research has been invested into extending these techniques towards in situ or operando characterization [1].

Here, we will present our recent work looking at the structural transformations of noble metal nanoparticles during $\mathrm{CO}$ oxidation, a prototypical catalytic reaction, with gas cell transmission electron microscopy. We will describe how the adsorption of $\mathrm{CO}$ molecules changes the surface facets of $\mathrm{Pd}$ and Pt nanoparticles, the model materials for $\mathrm{CO}$ oxidation [2], and how these changes correlate with their catalytic activity. In particular, we focus on the differences in morphology at temperatures where these nanoparticles exhibit $\mathrm{CO}$ poisoning versus temperatures where they show stable $\mathrm{CO}$ conversion at 1 bar pressure. As shown in Figure 1(a), the introduction of $\mathrm{CO}$ into the gas stream at $200{ }^{\circ} \mathrm{C}$ caused the surface facets of a $\mathrm{Pd}$ nanoparticle to flatten and the formation of sharp corners at the nanoparticle edges. These nanoparticles exhibited no activity until the temperature was raised to $500{ }^{\circ} \mathrm{C}$, where the corners disappeared as seen in Figure 1(b). When the temperature was lowered back to $300{ }^{\circ} \mathrm{C}$, these sharp features begun to reappear on the Pd nanoparticle surface and the activity dropped. The heating profiles and mass spectrometer results are presented in Figure 1(c) and Figure 1 (d).

It has been reported that Pt nanoparticles exhibit restructuring similar to what we described for Pd when they are in a bi-stable state where their activity oscillates [3]. We studied Pt nanoparticles under experimental conditions similar to that of the Pd nanoparticles (Figure 1(e)) and found that the degree of surface restructuring for Pt is less dramatic when compared to Pd.

References:

[1] A Chakrabarti et al., Catal. Today 283 (2017) p. 27.

[2] M.A. Van Spronsen, J.W.M Frenken, and I.M.N Groot, Chem. Soc. Rev. 46, (2017) p. 4347.

[3] S.B. Vendelbo et al, Nat. Mat. 13 (2014) p. 884.

[4] The authors acknowledge funding from the National Research Foundation of Singapore. 
(a) $\mathrm{Pd}, 200^{\circ} \mathrm{C}$
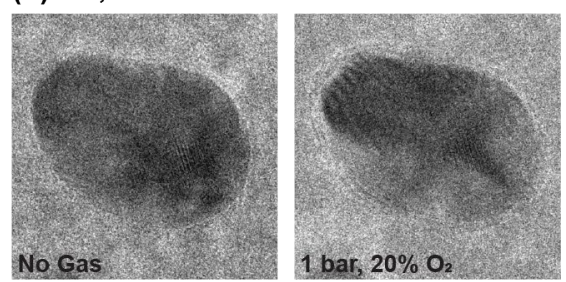

(b) Pd, 1 bar, 9\% CO, 18\% $\mathrm{O}_{2}$

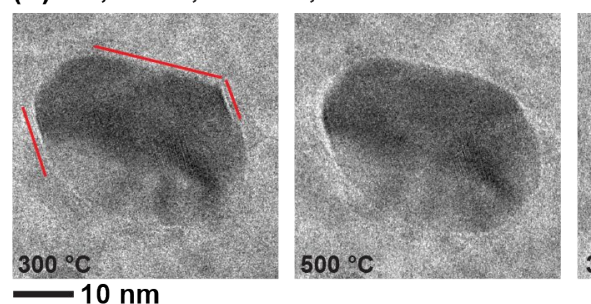

(e) Pt, 1 bar

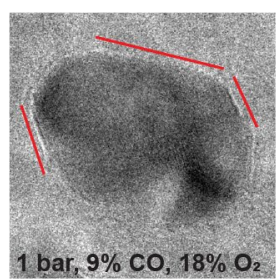

$200^{\circ} \mathrm{C}, 20 \% \mathrm{O}$
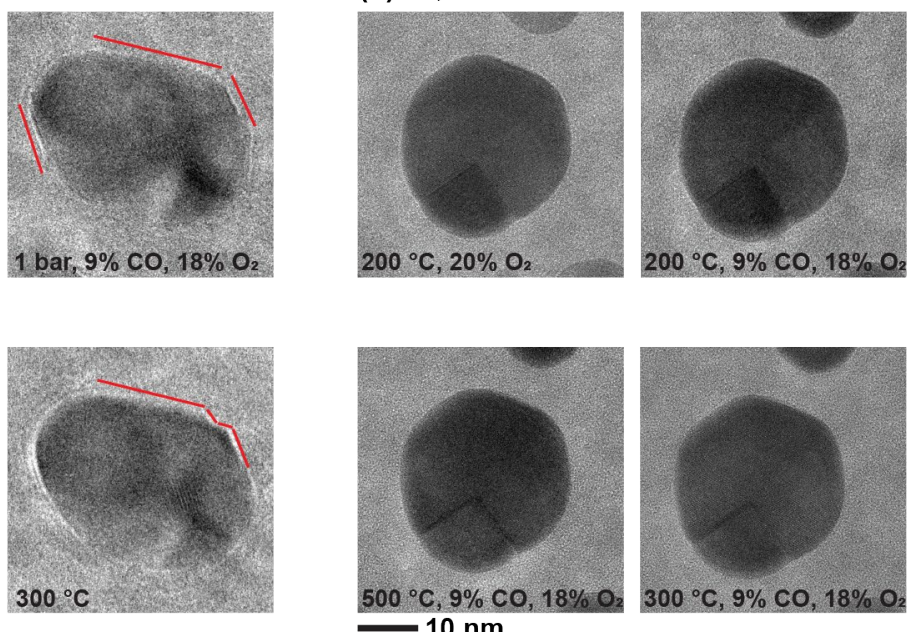

$500^{\circ} \mathrm{C}, 9 \% \mathrm{CO}, 18 \% \mathrm{O}_{2}, 300^{\circ} \mathrm{C}, 9 \% \mathrm{CO}, 18 \% \mathrm{O}$

(c)

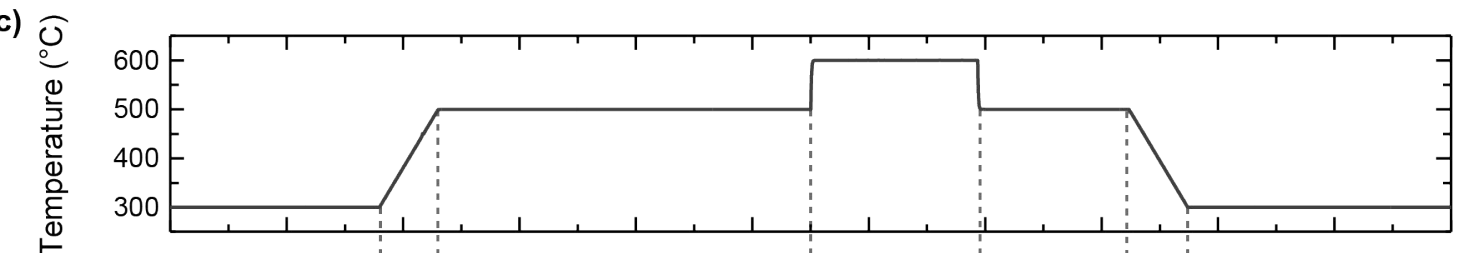

(d)

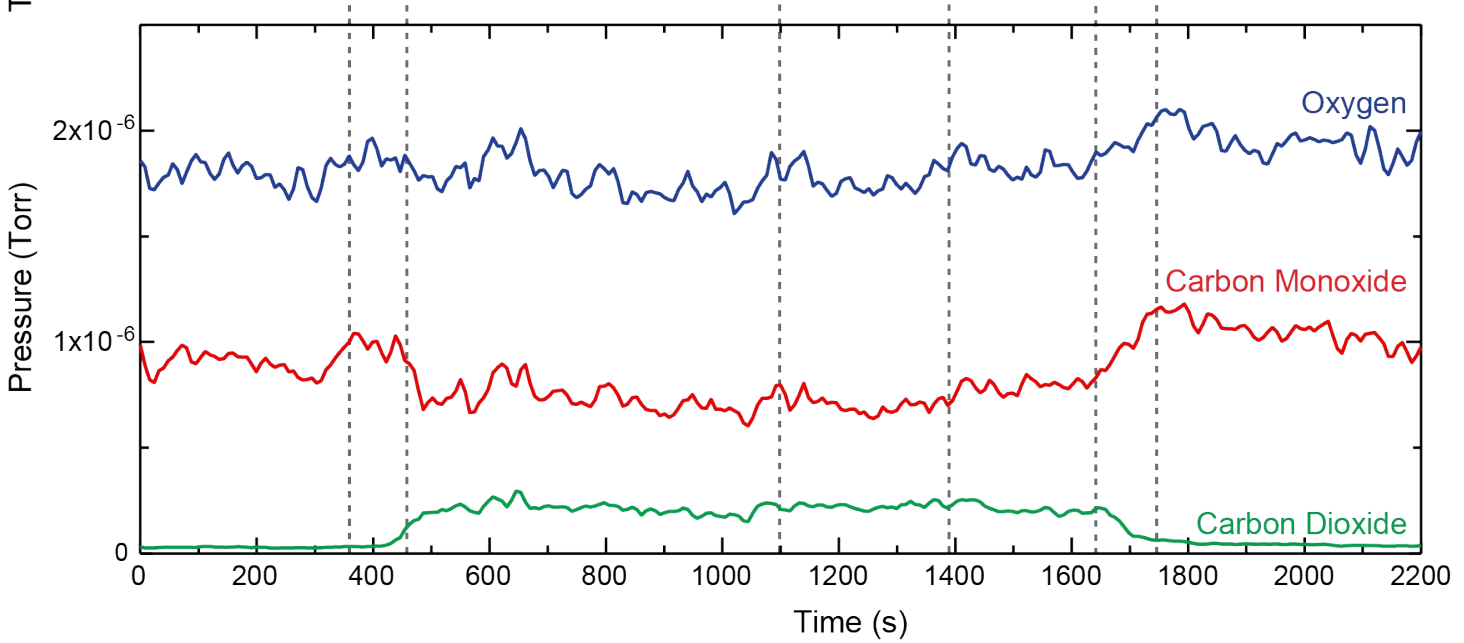

Figure 1. (a) TEM micrographs show the morphological changes in a Pd nanoparticle when $\mathrm{O}_{2}$ and $\mathrm{CO}$ (with He as carrier gas) is introduced in sequence into the gas stream at $200{ }^{\circ} \mathrm{C}$. At this temperature, the nanoparticles are $\mathrm{CO}$ poisoned. (b) TEM micrographs of the same nanoparticle where the temperature is changed from $300{ }^{\circ} \mathrm{C}$ to $500{ }^{\circ} \mathrm{C}$ and back to $300{ }^{\circ} \mathrm{C}$. The full temperature profile is shown in (c) and the gas compositions measured using an inline mass spectrometer are shown in (d) It is clear from (d) that the nanoparticles only be become active after the temperature is raised. In (e), we show TEM micrographs of a Pt nanoparticle under similar environmental conditions. 